\title{
Elevated expression of IL-10RB acts as an independent unfavorable prognostic marker and predicts the polarization of tumor-associated macrophages in glioma
}

\author{
Yaqiong Fan \\ Capital Medical University \\ Yichen Peng \\ Capital Medical University \\ Feng Chen \\ Capital Medical University \\ Wenbin Li ( $\sim$ liwenbin@ccmu.edu.cn ) \\ Capital Medical University
}

\section{Research Article}

Keywords: IL10RB, glioma, prognostic marker, macrophages, immunosuppressive

Posted Date: April 8th, 2021

DOI: https://doi.org/10.21203/rs.3.rs-364328/v1

License: (c) (1) This work is licensed under a Creative Commons Attribution 4.0 International License.

Read Full License 


\section{Abstract}

Background: Interleukin-10 receptor B (IL10RB) is a subunit of the interleukin-10 receptor. As an important part of the interleukin-10 signaling pathway, IL10RB plays an essential role in the regulation of the immune response. However, studies of IL10RB in glioma are rare. The present study aimed to investigate the potential role of IL10RB in glioma.

Methods: 611 glioma samples in TCGA dataset and 310 glioma samples in CGGA dataset were download and organized, then analyzed the clinical expression characteristics and prognostic value of IL10RB as well as its correlation with immune cell infiltration in these samples using R.

Results: IL10RB expression was significantly higher in the glioma tissues than in the normal tissues and increased in gliomas, especially with malignant phenotype. The Kaplan-Meier survival analysis and Cox regression analysis indicated that IL10RB was significantly associated with poor prognosis in patients with glioma and could be used as an independent prognostic marker. Further functional analysis suggested that IL10RB was involved in immune responses, and high expression of IL10RB in the glioma tissue resulted in more infiltrated immune cells, especially macrophages. Moreover, IL10RB was found to be strongly correlated with marker genes of tumor-associated macrophages (TAMs) and M2 macrophages, but low or even no correlation with marker genes of M1 macrophages. In addition, there was also a significant association between IL10RB and immune checkpoints that suppress effective antitumor immune responses.

Conclusions: Our results demonstrated that high expressed IL10RB was associated with the malignant progression of gliomas and poor prognosis, and it might be involved in the polarization of macrophages which play a crucial role in the immunosuppressive response of gliomas.

\section{Background}

Glioma occurs in neuroectodermal tissue and accounts for $81 \%$ of all primary central nervous system (CNS) malignant tumors according to statistics from the Central Brain Tumor Registry of the United States (CBTRUS)[1]. Despite the constant improvement in comprehensive therapy including neurosurgical resection, adjuvant radiotherapy, and temozolomide (TMZ) chemotherapy, patients with gliomas still have a short median survival time[2, 3] and a 5-year survival rate, which is only $9 \%-12 \%[4]$. It is tough to treat glioma because of its several traits including invasive growth, blood-brain barrier, drug resistance, as well as inherent heterogeneity. However, studies on the anticancer immune responses has made a breakthrough in the past decades, faciliting the immunotherapy becoming a novel treatment strategy for tumors[5]. Meanwhile, the discovery of lymphatic system in the CNS has provided a new theoretical basis and opportunity for the immunotherapy of intracranial tumors[6]. Increasingly immunological studies have been conducted on glioma in order to Identify immunotherapy targets and explore effective treatment[7, 8]. 
Glioma possesses a complex tumor microenvironment mainly consisting of tumor cells, immune cells, fibroblasts, nearby interstitial tissues, microvessels, and various cytokines and chemokines. It has been reported that the infiltrating immune cells such as microglia, tumor-associated macrophages (TAMs), regulatory T cells (Tregs), dendritic cells (DCs), and myeloid-derived suppressor cells (MDSCs) could create an immunosuppressive microenvironment to facilitate tumor growth, metastasis, angiogenesis as well as tumor immune escape[9-11]. As the most abundant immune cells, TAMs, in particular, is served as a significant regulator in the glioma microenvironment[12]. For instance, TAMs secrete cytokines such as IL-4, IL-10, and IL-13 to stimulate Th2 immune responses. TAMs can also secrete immunosuppressive factors TGF- $\beta$, IL-10, and prostaglandin E2 (PGE2) under the action of the chemokine CCL22 to recruit Treg cells, thereby inhibiting the anti-tumor response. Additionally, TAMs express programmed cell deathligand1 (PD-L1) to promote T cell apoptosis and participate in immunosuppression[13, 14]. Hence, a detailed understanding of the immune microenvironment of glioma is essential for developing new immunotherapeutic strategies.

IL-10 is an anti-inflammatory and immunosuppressive cytokine, mainly derived from mononuclear / macrophages and T helper cells. The multiple activities of IL-10 are mediated by specific cell surface receptor complexes. Studies have shown that IL-10 cytokine receptor (IL-10R) is a transmembrane protein that including IL-10RA and IL-10RB receptor chains. IL-10 binds to IL-10RA with high affinity and then binds to IL-10RB with lower affinity to form an IL10 signal transduction complex[15, 16]. After being polymerized, the receptor transmembrane molecule can activate JAK1 kinase and Tyk2 kinase which influence thephosphorylation of STAT factors, thereby affecting the transcription of target genes and exerting various regulatory effects of IL-10[17]. Several pieces of evidence showed that the IL-10 signal transduction complex could prevent the maturation of DCs and increas the differentiation of macrophages, resulting in the reduced antigen presentation and enhanced immune suppression. In addition, IL-10 inhibits the release of cytotoxic cytokine IFN- $\gamma$, stimulates the differentiation of ineffective T cells, and promotes tumor immune escape in TME[18]. Moreover, researchers found that glioma-derived factors can indirectly increase the expression of PD-L1 in macrophages by enhancing autocrine/paracrine IL-10 signaling, thereby promoting immunosuppressive effects in the local microenvironment and systemic circulation[19]. Unfortunately, as an important part of the IL-10 signal transduction complex, IL10RB has been rarely studied in gliomas. In our study, we managed to conduct a comprehensive analysis of IL10RB in gliomas based on two independent RNA sequencing databases, The Cancer Genome Atlas (TCGA) and the Chinese Glioma Genome Atlas (CGGA). We investigated IL10RB expression patterns and prognostic value in glioma patients and further explored its functional roles. As shown in the results, IL10RB expression was significantly higher in glioma, compared with normal tissues, and increased with the tumor grade. Elevated IL10RB associated with poor prognosis of glioma patients. Furthermore, IL10RB expression showed a positive correlation with tumor infiltration of M2 macrophages and predicted the polarization of TAMs in glioma. Collectively, these results allow us to contribute to current research on the potential role of IL10RB in gliomas.

\section{Methods}




\section{Patients and data collection}

611 samples (214 grade II, 237 grade III, and 160 grade IV gliomas, http://cancergenome.nih.gov/) in TCGA dataset and 310 samples (105 grade II, 67 grade III, and 138 grade IV gliomas, http://www.cgga.org.cn) in CGGA dataset were analyzed in our study. The clinical and molecular characteristics of the selected patients are detailed in Table S1.

\section{Statistical analysis}

All statistical analyses and graph generation were performed with $R$ software ( $R$ version 4.0.3; https://www.r-project.org/). The protein expression of IL10RB in gliomas and normal tissues by immunohistochemistry image using The Human Protein Atlas (HPA) (https://www.proteinatlas.org/), a freely accessible website. A p-value of less than 0.05 was considered statistically significant. The student's t-test was conducted to compare the differences between two different IL10RB expression levels. The contribution IL10RB made to survival was revealed by Kaplan-Meier survival analysis and the log-rank test. Time-dependent receiver operating characteristic (ROC) curve analysis was used to predict 1-, 3- and 5-year overall survival (OS). The univariable and multivariable Cox regression analyses were applied to evaluate the value of IL10RB in prognosis, and the forest plots were made by the "forestplot" package. Pearson's correlation analysis was performed to assess the correlation between IL10RB and other related genes. The immune score and tumor purity were calculated by the ESTIMATE package, and the immune cell infiltration in samples was evaluated by CIBERSORT (https://cibersort stanford.edu/).

\section{Results}

\section{IL10RB expression increased the malignancy of gliomas}

To clarify the characteristics of IL10RB in gliomas, we first evaluated the differences of IL10RB expression levels in the TCGA and CCGA datasets based on tumor grade, isocitrate dehydrogenase (IDH) mutation status, and 1p/19q coding status. As exhibited in Fig. 1, the analyses for the IL10RB expression level in the TCGA and CCGA datasets shared a similar conclusion. IL10RB expression increased significantly along with tumor grade and reached the highest in GBM (glioma grade IV) (Fig. 1A, D). In both LGG and GBM, IL10RB expression was higher in the IDH wild-type (IDH-wt) group than the IDH mutant-type (IDH-mut) group (Fig. 1B, C for TGGA, Fig. 1E, F for CCGA). The expression of IL10RB in different glioma grades was further confirmed by immunohistochemical staining (Fig. 1G), which also suggested that the high level of IL10RB expression was associated with malignant progression of glioma.

Next, in order to have a deeper understanding of the role IL10RB takes in glioma, we further analyzed the IL10RB expression of four glioma subtypes in the TCGA and CGGA datasets. The results indicated that the mesenchymal subtype exhibited the strongest expression of IL10RB, successively followed by the neural, classical, and proneural subtypes in the TCGA dataset (Fig. 2A). Although the analysis results based on the CGGA dataset are slightly different from TCGA, it still suggests that IL 10RB is highly expressed in mesenchymal subtype gliomas (Fig. 2C). Gliomas were next divided into mesenchymal and 
non-mesenchymal subtype groups for ROC curve analysis, and the area under the curve (AUC) was 0.879 and 0.918 for the TCGA and CGGA cohorts, respectively (Fig. 2B, D), suggesting that IL10RB expression levels could be highly efficient in distinguishing mesenchymal subtypes from the rest three subtypes. It's becoming apparent that the mesenchymal subtypes have an important role in elevating tumor malignancy[20]. Altogether, the results demonstrated there was a strong correlation between the increasing IL10RB expression and glioma malignancy.

\section{High IL10RB expression could be an independent indicator for poor outcomes}

Knowing the association between up-regulated IL10RB expression and tumor malignancy, we further evaluated the contribution of IL10RB expression level made in prognosis. Kaplan-Meier survival analyses were performed separately for LGG and GBM by applying the median IL10RB expression level as a cutoff, and the results showed that patients who suffered from either LGG or GBM would have a reduced OS for the high IL10RB expression (Fig. 3A-D). Next, the specificity and sensitivity in AUC of IL10RB expression were compared with age at diagnosis and tumor grade in predicting the overall survival (OS), and as is showed in Fig. 3E, the IL10RB expression was more predictive than the other two with 1-, 3-, and 5-year OS were $0.733,0.832$, and 0.811 (Fig. 3E).

Furthermore, the univariable and multivariable Cox analyses were conducted to ensure if IL10RB expression was an independent prognostic biomarker for gliomas. After conducting multivariable Cox analysis of variables in Fig. 4 and Figure S1, we noticed that the IL10RB expression still tightly regulated the OS in the CGGA and TCGA data set.

All in all, the results above confirmed that not only a high expression of IL10RB was positively correlated with the poor prognosis of patients with gliomas, but also IL10RB itself was an independent, potential prognostic biomarker.

\section{IL10RB was correlated with immune functions in gliomas}

To further explore the effect of IL10RB on gliomas, we used Pearson's correlation analysis to analyze the genes that are closely related to IL10RB $(R \geq 0.5)$ in the TGGA and CGGA datasets, respectively. The genes positively related to IL10RB expression are shown in Fig. 5A and Figure S2. Furthermore, the positive genes to IL10RB expression Using $R$ packages to perform functional annotation analysis on these two positively related genomes, we found that these genes are mainly involved in immune response, inflammatory response, extracellular organization, leukocyte migration, antigen processing, and presentation (Fig. 5B). These results indicated that IL10RB is closely related to immune response, and the over-expression of IL10RB may promote gliomas progression through extracellular matrix organization and angiogenesis.

\section{IL10RB could predict the polarization of tumor-associated macrophages and was associated with immunosuppressive phenotype in gliomas}

To further investigate the close correlation between IL10RB and immune responses in glioma, we applied the ESTIMATE algorithm to assess the relationship between IL10RB and immune infiltration [21]. The 
elevated IL10RB expression levels significantly led to the increased immune scores and the decreased tumor purity, which was consistent in TGGA and CCGA datasets (Fig. 6A). Next, we evaluated the abundance of a variety of immune cell types in the TGGA and CCGA datasets by using the CIBERSORT [22]. A great number of immune cells were correlated with the high IL10RB expression, especially Macrophages M2 in the TCGA dataset and Macrophages M0 in the CGGA dataset (Fig. 6B). It was likely that glioma samples with higher IL10RB expression infiltrated with more macrophages, then this speculation was confirmed by Fig. 7A which showed a strong positive correlation between IL10RB and macrophages in both TCGA and CGGA datasets [23].

TAMs in the glioma microenvironment is consist of infiltrated peripheral monocytes and resident microglia in the central nervous system. TAMs can be converted between two phenotypes, M1 and M2, by multiple cytokines in the glioma microenvironment. In order to explore the relationship between IL10RB and macrophages for the 2 phenotypes exhibiting opposite effects on tumor progression [24], we further analyzed the correlation between IL10RB and characteristic marker genes of TAMs, M1, and M2 [25]. As is showed in Fig. 7B, there was a significant correlation between IL10RB and M2 macrophages rather than M1 macrophages.

Finally, we analyzed the association of IL10RB expression level with some vital immune checkpoints which can represent the tumor immune microenvironment and can be used as a potential therapeutic target. As illustrated in Fig. 7C, IL10RB expression was highly positively correlated to the checkpoints that suppress the antitumor immune response, including PD-1, PD-L2, T cell immunoglobulin and mucin domain-3 (TIM-3), lymphocyte activation gene-3 (LAG3), indoleamine 2,3-dioxygenase 1 (IDO1) and inducible T cell costimulatory (ICOS) [26]. This result suggested that IL10RB may play an important role in the treatment of immune checkpoint inhibitors.

\section{Discussion}

Glioma is the most common primary intracranial tumor and has a high mortality rate[27]. Traditional surgery combined with radiotherapy and chemotherapy did not significantly improve the prognosis of glioma patients, making the new clinical therapeutic methods to be urgently needed.

Studies have shown that tumor immune microenvironment plays a vital role in tumorigenesis and progression, giving rise to the emerging immunotherapy which has achieved marked success in the clinical treatment of tumors. For instance, cellular immunotherapy as chimeric antigen receptor $\mathrm{T}$ cell therapy (CAR-T) has a significant effect on patients with lymphoma and leukemia $[28,29]$. The immune checkpoint inhibitors, especially monoclonal antibodies against PD-1/PDL-1 and CTLA-4 have been approved by the FDA for the first-line treatment of melanoma [30], non-small-cell lung cancer (NSCLC) [31], renal cancer [32] as well as head and neck cancer [33].

Additionally, a growing number of immunotherapeutics including peptide vaccines [34], dendritic cell vaccines [35], immunovirotherapy and cytokine therapy [36] are under clinical investigation for their safety and efficacy. 
However, the response rates of many immunotherapy methods for glioma are low due to the special immune microenvironment of the CNS. To break the limitations of immunotherapy for glioma, researchers continue to make attempt The failure of the anti-tumor effect with single-agent or single-cell immunotherapy has prompted the multidisciplinary treatment of glioma and the optimal combination of all possible treatment methods. It is well known that macrophages are polarized into two subsets, M2 and M1. Among them, M1 macrophages secrete Th1 cytokines, such as IL-6, 8,12, and tumor necrosis factor (TNF)-a, leading to primarily anti-cancer responses. However, M2 macrophages produce Th2 cytokines such as IL-4, IL-10, and IL-13 to promote the proliferation of Th2 cells and induce immune tolerance by activation of Treg cells. Reducing recruitment of TAMs into the tumor microenvironment and fostering repolarization of M2 to M1 phenotype are being studied as new therapeutic strategies. Moreover, cytokine-based immunomodulators are being developed as adjuvant treatment approaches by correcting imbalanced immune function, improving immune suppression, and enhancing anti-tumor immune response [37].

IL10 is a pleiotropic cytokine mainly secreted by antigen-presenting cells such as activated T cells, B cells, monocytes, and macrophages, which can affect the activities of various cell types in the immune system. The immunomodulatory function of IL10 is mediated by the transmembrane signal transduction complex composed of IL10RA and IL10RB. Studies have shown that IL10 is associated with the survival, proliferation, and anti-apoptotic activity of various cancers such as Burkitt's lymphoma [38], nonHodgkin's lymphoma [39], and non-small cell lung cancer [40]. Targeting tumors with IL-10 not only prevents dendritic cell-mediated CD8 + T cell apoptosis, but also significantly improves antitumor effects in mice with advanced tumors when combined with immune checkpoint blockade (CmAb-(IL10)2) [41]. Drugs related to IL10, such as IL10 fusion protein, polyethylene glycosylated IL10 (pegilodecakin) [42]and adenovirus are in the clinical research stage, and initially demonstrated good anti-tumor effects [43]. However, with the increasing research of IL-10 in tumor diseases, its role in the occurrence and development of different tumors has also been controversial. Especially, there are very few studies on IL10 and its signaling pathways in gliomas. Here, through a preliminary exploration of the database, we screened the close relationship between IL10RB and glioma, resulting in a comprehensive analysis of the expression, prognostic value, and function of IL10RB in glioma. According to our results, the high expression levels of IL10RB showed an association with the malignant phenotype, such as high WHO Grade, the IDH wildtype status, and mesenchymal subtype in gliomas. Critically, elevated IL10RB expression predicted significantly worse survival in glioma patients. We found that the high IL10RB expression was shown positively related to inflammatory activities and immune responses in whole gliomas, and glioma tissues with higher IL10RB expression are infiltrated with more microglia/macrophages. Further analysis indicated that IL10RB can promote the polarization of TAMs to protumoral M2 type. Meanwhile, we confirmed the strong correlation between IL10RB and some important immune checkpoints as PD-1, PD-L2, T cell immunoglobulin and mucin domain-3 (TIM-3), lymphocyte activation gene-3 (LAG3), indoleamine 2,3-dioxygenase 1 (ID01), and inducible T cell costimulatory (ICOS). For these glioma patients, targeting IL10 and (or) IL10RB may be able to reduce 
immunosuppression through macrophage repolarization and synergistically enhance the therapeutic effect of immune checkpoint inhibitors.

Taken together, through the comprehensive bioinformatics analysis of IL10RB, we found that IL10RB was upregulated in high malignant glioma, and was an independent indicator for the clinical prognosis of glioma patients. Importantly, IL10RB was positively related to the infiltration of immune cells in the glioma microenvironment, especially TAMs and could predict the polarization of tumor-associated macrophages. These findings indicated IL 10RB is a novel potential target for enhancing the anticancer therapies, and its value in clinical immunotherapy for glioma patients is worth a further study.

The limitations of this study are several. First, the public data source is a combination of multiple centers, the lack of partial data and the differences in data collection and treatment methods might directly lead to the deviation of analysis results.Secondly, performing the in vitro or/and in vivo experiments to verify our results would also make a difference.

\section{Conclusion}

We identified a gene IL-10RB, which acts as an independent unfavorable prognostic marker and predicts the polarization of tumor-associated macrophages in glioma. IL10RB could be used as a potential target for immunotherapy of glioma.

\section{Abbreviations}

GBM: Glioblastoma multiforme; OS: Overall survival; TCGA: The Cancer Genome Atlas; CGGA: Chinese Glioma Genome Atlas; IDH: isocitrate dehydrogenase; MGMT: methylguanine methyltransferase; GO: Gene ontology; ROC: Receiver operating characteristic; AUC: Area under the ROC; TME: Tumor microenvironment; TAMs: Tumor-associated macrophages

\section{Declarations}

\section{Ethics approval and consent to participate}

Not applicable. All data in this study are publicly available.

\section{Consent for publication}

Not applicable.

\section{Availability of data and materials}

The datasets analyzed during the current study are available in the TCGA (http://cancergenome.nih.gov/) and CGGA ( http://www.cgga.org.cn/) repository. All analyzed data are included in this article and its 
supplementary information file. The original data are available from the corresponding author on reasonable request.

\section{Competing interests}

The authors declare that there are no conflicts of interest.

\section{Funding}

This work was supported by grants from the National Natural Science Foundation of China (No.81972338); Beijing Municipal Science \& Technology Commission (Z181100001718127).

\section{Authors' contribution}

YQF conceived the study, downloaded and organized the clinical and gene expression data and performed the bioinformatics analyses. YQF and YCP wrote the original draft. FC revised the article for essential intellectual content. WBL provided supervision and administrative support. All authors read and approved the final manuscript.

\section{Acknowledgment}

Not applicable.

\section{Author details}

${ }^{1}$ Beijing Tiantan Hospital, Capital Medical University, Beijing 100050, PR China.

${ }^{2}$ Department of Neuro-Oncology, Neurosurgery Center, Beijing Tiantan Hospital, Capital Medical University, Beijing 100050, PR China.

\section{Authors' information}

Not applicable.

\section{References}

1. Ostrom QT, Gittleman H, Truitt G, Boscia A, Kruchko C, Barnholtz-Sloan JS. CBTRUS Statistical Report: Primary Brain and Other Central Nervous System Tumors Diagnosed in the United States in 2011-2015. Neuro Oncol. 2018;20:iv1-1iv86.

2. Jiang T, Mao Y, Ma W, Mao Q, You Y, Yang X, Jiang C, Kang C, Li X, Chen L, Qiu X, Wang W, Li W, Yao Y, Li S, Li S, Wu A, Sai K, Bai H, Li G, Chen B, Yao K, Wei X, Liu X, Zhang Z, Dai Y, Lv S, Wang L, Lin Z, Dong J, Xu G, Ma X, Cai J, Zhang W, Wang H, Chen L, Zhang C, Yang P, Yan W, Liu Z, Hu H, Chen J, Liu Y, Yang Y, Wang Z, Wang Z, Wang Y, You G, Han L, Bao Z, Liu Y, Wang Y, Fan X, Liu S, Liu X, Wang Y, 
Wang Q. CGCG clinical practice guidelines for the management of adult diffuse gliomas. Cancer Lett. 2016;375:263-73.

3. Yang P, Wang Y, Peng X, You G, Zhang W, Yan W, Bao Z, Wang Y, Qiu X, Jiang T. Management and survival rates in patients with glioma in China (2004-2010): a retrospective study from a singleinstitution. J Neurooncol. 2013;113:259-66.

4. Stupp R, Hegi ME, Mason WP, van den Bent MJ, Taphoorn MJ, Janzer RC, Ludwin SK, Allgeier A, Fisher B, Belanger K, Hau P, Brandes AA, Gijtenbeek J, Marosi C, Vecht CJ, Mokhtari K, Wesseling P, Villa S, Eisenhauer E, Gorlia T, Weller M, Lacombe D, Cairncross JG, Mirimanoff RO. Effects of radiotherapy with concomitant and adjuvant temozolomide versus radiotherapy alone on survival in glioblastoma in a randomised phase III study: 5-year analysis of the EORTC-NCIC trial. Lancet Oncol. 2009;10:459-66.

5. Rusch T, Bayry J, Werner J, Shevchenko I, Bazhin AV. Immunotherapy as an Option for Cancer Treatment. Arch Immunol Ther Exp (Warsz). 2018;66:89-96.

6. Louveau A, Smirnov I, Keyes TJ, Eccles JD, Rouhani SJ, Peske JD, Derecki NC, Castle D, Mandell JW, Lee KS, Harris TH, Kipnis J. Structural and functional features of central nervous system lymphatic vessels. Nature. 2015;523:337-41.

7. Parney IF, Hao C, Petruk KC. Glioma immunology and immunotherapy. Neurosurgery. 2000;46:778-91; discussion 791-2.

8. Preusser M, Lim M, Hafler DA, Reardon DA, Sampson JH. Prospects of immune checkpoint modulators in the treatment of glioblastoma. Nat Rev Neurol. 2015;11:504-14.

9. Domingues P, González-Tablas M, Otero Á, Pascual D, Miranda D, Ruiz L, Sousa P, Ciudad J, Gonçalves JM, Lopes MC, Orfao A, Tabernero MD. Tumor infiltrating immune cells in gliomas and meningiomas. Brain Behav Immun. 2016;53:1-15.

10. Gieryng A, Pszczolkowska D, Walentynowicz KA, Rajan WD, Kaminska B. Immune microenvironment of gliomas. Lab Invest. 2017;97:498-518.

11. Quail DF, Joyce JA. The Microenvironmental Landscape of Brain Tumors. Cancer Cell. 2017;31:32641.

12. Chanmee T, Ontong P, Konno K, Itano N. Tumor-associated macrophages as major players in the tumor microenvironment. Cancers (Basel). 2014;6:1670-90.

13. Noy R, Pollard JW. Tumor-associated macrophages: from mechanisms to therapy. Immunity. 2014;41:49-61.

14. Hambardzumyan $\mathrm{D}$, Gutmann $\mathrm{DH}$, Kettenmann $\mathrm{H}$. The role of microglia and macrophages in glioma maintenance and progression. Nat Neurosci. 2016;19:20-7.

15. Liu Y, Wei SH, Ho AS, de Waal Malefyt R, Moore KW. Expression cloning and characterization of a human IL-10 receptor. J Immunol. 1994;152:1821-9.

16. Moore KW, de Waal Malefyt R, Coffman RL, O\&\#39, Garra A. Interleukin-10 and the interleukin-10 receptor. Annu Rev Immunol. 2001;19:683-765. 
17. Finbloom DS, Winestock KD. IL-10 induces the tyrosine phosphorylation of tyk2 and Jak1 and the differential assembly of STAT1 alpha and STAT3 complexes in human T cells and monocytes. $J$ Immunol. 1995;155:1079-90.

18. Ben-Baruch A. Inflammation-associated immune suppression in cancer: the roles played by cytokines, chemokines and additional mediators. Semin Cancer Biol. 2006;16:38-52.

19. Bloch O, Crane CA, Kaur R, Safaee M, Rutkowski MJ, Parsa AT. Gliomas promote immunosuppression through induction of B7-H1 expression in tumor-associated macrophages. Clin Cancer Res. 2013;19:3165-75.

20. Chen Z, Wang H, Zhang Z, Xu J, Qi Y, Xue H, Gao Z, Zhao R, Wang S, Zhang S, Qiu W, Guo X, Li G. Cell surface GRP78 regulates BACE2 via lysosome-dependent manner to maintain mesenchymal phenotype of glioma stem cells. J Exp Clin Cancer Res. 2021;40:20.

21. Yoshihara K, Shahmoradgoli M, Martínez E, Vegesna R, Kim H, Torres-Garcia W, Treviño V, Shen H, Laird PW, Levine DA, Carter SL, Getz G, Stemke-Hale K, Mills GB, Verhaak RG. Inferring tumour purity and stromal and immune cell admixture from expression data. Nat Commun. 2013;4:2612.

22. Newman AM, Liu CL, Green MR, Gentles AJ, Feng W, Xu Y, Hoang CD, Diehn M, Alizadeh AA. Robust enumeration of cell subsets from tissue expression profiles. Nat Methods. 2015;12:453-7.

23. Racle J, de Jonge K, Baumgaertner P, Speiser DE, Gfeller D. Simultaneous enumeration of cancer and immune cell types from bulk tumor gene expression data. Elife. 2017;6.

24. Mantovani A, Sozzani S, Locati M, Allavena P, Sica A. Macrophage polarization: tumor-associated macrophages as a paradigm for polarized M2 mononuclear phagocytes. Trends Immunol. 2002;23:549-55.

25. Wu X, Qu D, Weygant N, Peng J, Houchen CW. Cancer Stem Cell Marker DCLK1 Correlates with Tumorigenic Immune Infiltrates in the Colon and Gastric Adenocarcinoma Microenvironments. Cancers (Basel). 2020;12.

26. Burugu S, Dancsok AR, Nielsen TO. Emerging targets in cancer immunotherapy. Semin Cancer Biol. 2018;52:39-52.

27. Ostrom QT, Gittleman H, Stetson L, Virk S, Barnholtz-Sloan JS. Epidemiology of Intracranial Gliomas. Prog Neurol Surg. 2018;30:1-11.

28. Neelapu SS, Locke FL, Bartlett NL, Lekakis LJ, Miklos DB, Jacobson CA, Braunschweig I, Oluwole OO, Siddiqi T, Lin Y, Timmerman JM, Stiff PJ, Friedberg JW, Flinn IW, Goy A, Hill BT, Smith MR, Deol A, Farooq U, McSweeney P, Munoz J, Avivi I, Castro JE, Westin JR, Chavez JC, Ghobadi A, Komanduri KV, Levy R, Jacobsen ED, Witzig TE, Reagan P, Bot A, Rossi J, Navale L, Jiang Y, Aycock J, Elias M, Chang D, Wiezorek J, Go WY. Axicabtagene Ciloleucel CAR T-Cell Therapy in Refractory Large B-Cell Lymphoma. N Engl J Med. 2017;377:2531-44.

29. Maude SL, Laetsch TW, Buechner J, Rives S, Boyer M, Bittencourt H, Bader P, Verneris MR, Stefanski HE, Myers GD, Qayed M, De Moerloose B, Hiramatsu H, Schlis K, Davis KL, Martin PL, Nemecek ER, Yanik GA, Peters C, Baruchel A, Boissel N, Mechinaud F, Balduzzi A, Krueger J, June CH, Levine BL, Wood P, Taran T, Leung M, Mueller KT, Zhang Y, Sen K, Lebwohl D, Pulsipher MA, Grupp SA. 
Tisagenlecleucel in Children and Young Adults with B-Cell Lymphoblastic Leukemia. N Engl J Med. 2018;378:439-48.

30. Wolchok JD, Chiarion-Sileni V, Gonzalez R, Rutkowski P, Grob JJ, Cowey CL, Lao CD, Wagstaff J, Schadendorf D, Ferrucci PF, Smylie M, Dummer R, Hill A, Hogg D, Haanen J, Carlino MS, Bechter O, Maio M, Marquez-Rodas I, Guidoboni M, McArthur G, Lebbé C, Ascierto PA, Long GV, Cebon J, Sosman J, Postow MA, Callahan MK, Walker D, Rollin L, Bhore R, Hodi FS, Larkin J. Overall Survival with Combined Nivolumab and Ipilimumab in Advanced Melanoma. N Engl J Med. 2017;377:134556.

31. Reck M, Rodríguez-Abreu D, Robinson AG, Hui R, Csőszi T, Fülöp A, Gottfried M, Peled N, Tafreshi A, Cuffe S, O\&\#39, Brien M, Rao S, Hotta K, Leiby MA, Lubiniecki GM, Shentu Y, Rangwala R, Brahmer JR. Pembrolizumab versus Chemotherapy for PD-L1-Positive Non-Small-Cell Lung Cancer. N Engl J Med. 2016;375:1823-33.

32. Motzer RJ, Escudier B, McDermott DF, George S, Hammers HJ, Srinivas S, Tykodi SS, Sosman JA, Procopio G, Plimack ER, Castellano D, Choueiri TK, Gurney H, Donskov F, Bono P, Wagstaff J, Gauler TC, Ueda T, Tomita Y, Schutz FA, Kollmannsberger C, Larkin J, Ravaud A, Simon JS, Xu LA, Waxman IM, Sharma P. Nivolumab versus Everolimus in Advanced Renal-Cell Carcinoma. N Engl J Med. 2015;373:1803-13.

33. Ferris RL, Blumenschein G Jr, Fayette J, Guigay J, Colevas AD, Licitra L, Harrington K, Kasper S, Vokes EE, Even C, Worden F, Saba NF, Iglesias Docampo LC, Haddad R, Rordorf T, Kiyota N, Tahara M, Monga M, Lynch M, Geese WJ, Kopit J, Shaw JW, Gillison ML. Nivolumab for Recurrent SquamousCell Carcinoma of the Head and Neck. N Engl J Med. 2016;375:1856-67.

34. Yamaguchi Y, Yamaue H, Okusaka T, Okuno K, Suzuki H, Fujioka T, Otsu A, Ohashi Y, Shimazawa R, Nishio K, Furuse J, Minami H, Tsunoda T, Hayashi Y, Nakamura Y. Guidance for peptide vaccines for the treatment of cancer. Cancer Sci. 2014;105:924-31.

35. Anguille S, Smits EL, Lion E, van Tendeloo VF, Berneman ZN. Clinical use of dendritic cells for cancer therapy. Lancet Oncol. 2014;15:e257-67.

36. Nagarsheth N, Wicha MS, Zou W. Chemokines in the cancer microenvironment and their relevance in cancer immunotherapy. Nat Rev Immunol. 2017;17:559-72.

37. Iwami K, Natsume A, Wakabayashi T. Cytokine Therapy of Gliomas. Prog Neurol Surg. 2018;32:7989.

38. Xu L, Wang X, Wang J, Liu D, Wang Y, Huang Z, Tan H. Hypoxia-induced secretion of IL-10 from adipose-derived mesenchymal stem cell promotes growth and cancer stem cell properties of Burkitt lymphoma. Tumour Biol. 2016;37:7835-42.

39. Blay JY, Burdin N, Rousset F, Lenoir G, Biron P, Philip T, Banchereau J, Favrot MC. Serum interleukin10 in non-Hodgkin's lymphoma: a prognostic factor. Blood. 1993;82:2169-74.

40. Vahl JM, Friedrich J, Mittler S, Trump S, Heim L, Kachler K, Balabko L, Fuhrich N, Geppert Cl, Trufa DI, Sopel N, Rieker R, Sirbu H, Finotto S. Interleukin-10-regulated tumour tolerance in non-small cell lung cancer. Br J Cancer. 2017;117:1644-55. 
41. Qiao J, Liu Z, Dong C, Luan Y, Zhang A, Moore C, Fu K, Peng J, Wang Y, Ren Z, Han C, Xu T, Fu YX. Targeting Tumors with IL-10 Prevents Dendritic Cell-Mediated CD8(+) T Cell Apoptosis. Cancer Cell. 2019;35:901-15.e4.

42. Autio K, Oft M. Pegylated Interleukin-10: Clinical Development of an Immunoregulatory Cytokine for Use in Cancer Therapeutics. Curr Oncol Rep. 2019;21:19.

43. Wang X, Wong K, Ouyang W, Rutz S. Targeting IL-10 Family Cytokines for the Treatment of Human Diseases. Cold Spring Harb Perspect Biol. 2019;11.

\section{Figures}



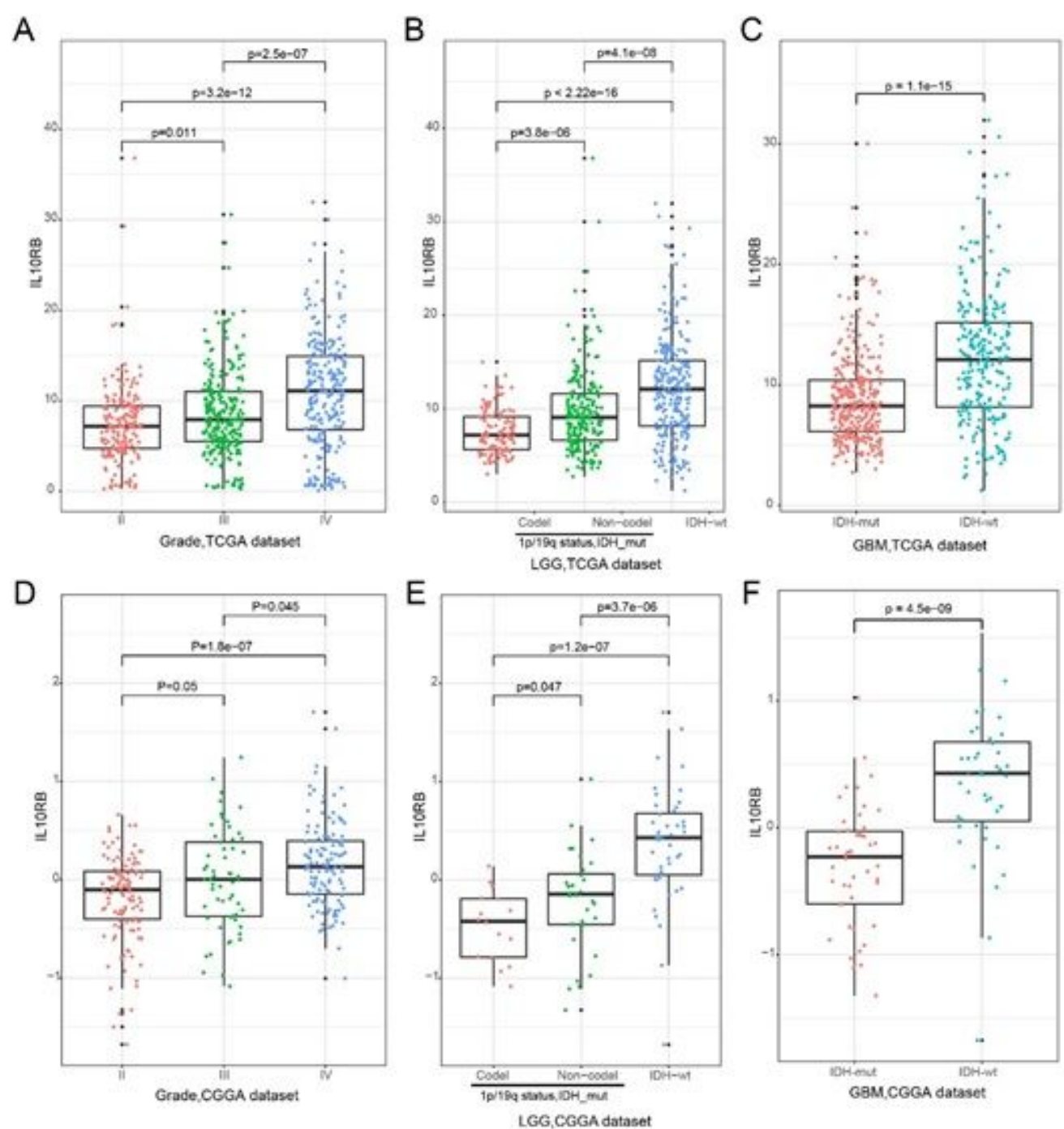

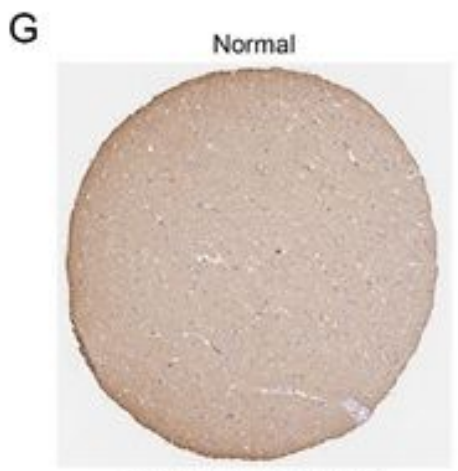

Staining: Not detected

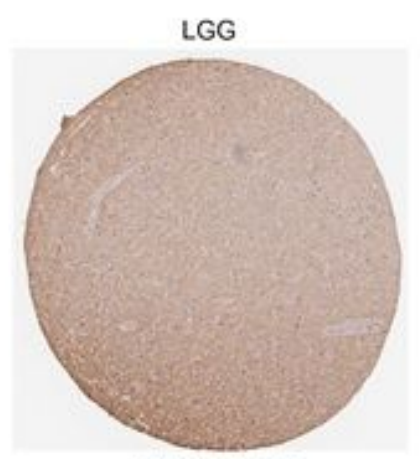

Staining: Low

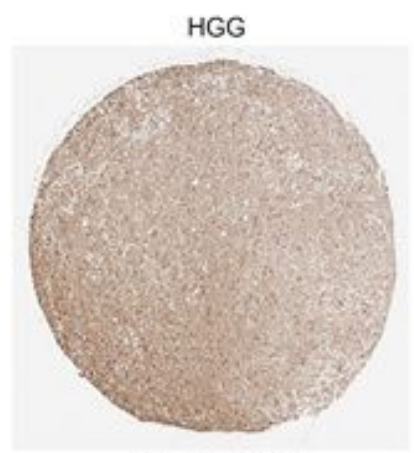

Staining: High

Antibody: HPA047550

\section{Figure 1}

IL10RB expression in different grouping methods of gliomas in TCGA and CGGA datasets. IL10RB expression based on the glioma grade (A and D). IL10RB expression with different IDH mutation status and $1 p / 19 q$ code deletion status in LGG (B and E). IL10RB expression in patients with GBM was stratified based on IDH mutation status ( $C$ and F). Immunostaining images of IL10RB in glioma samples based on glioma grades. 


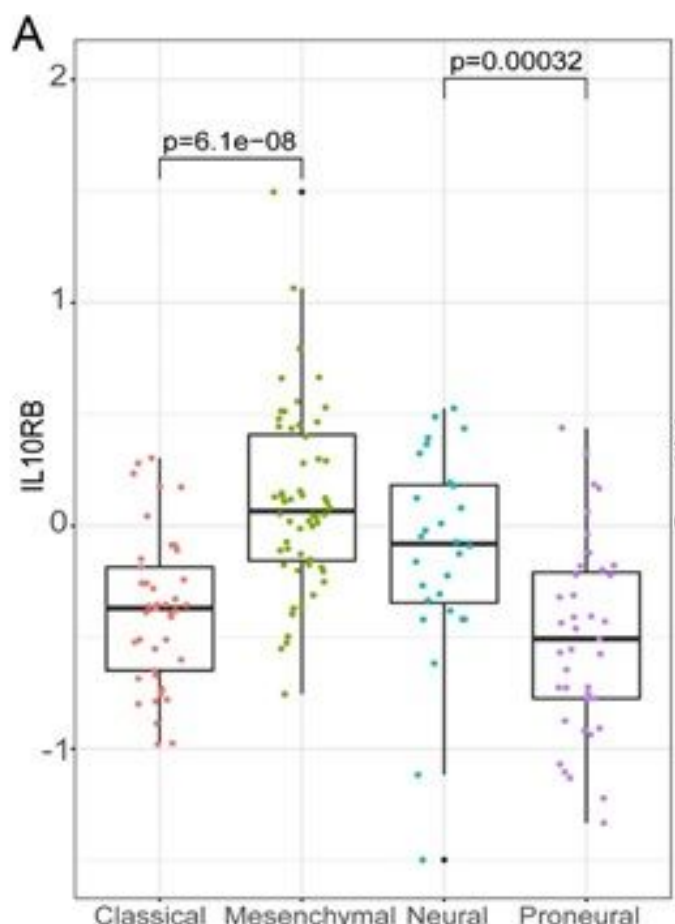

Subtype,TCGA dataset

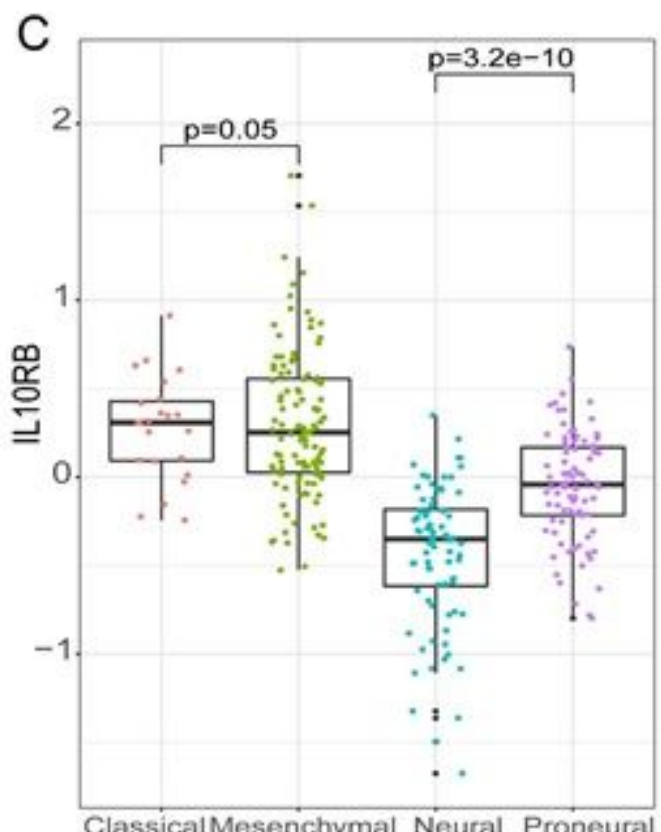

Subtypes,CGGA dataset
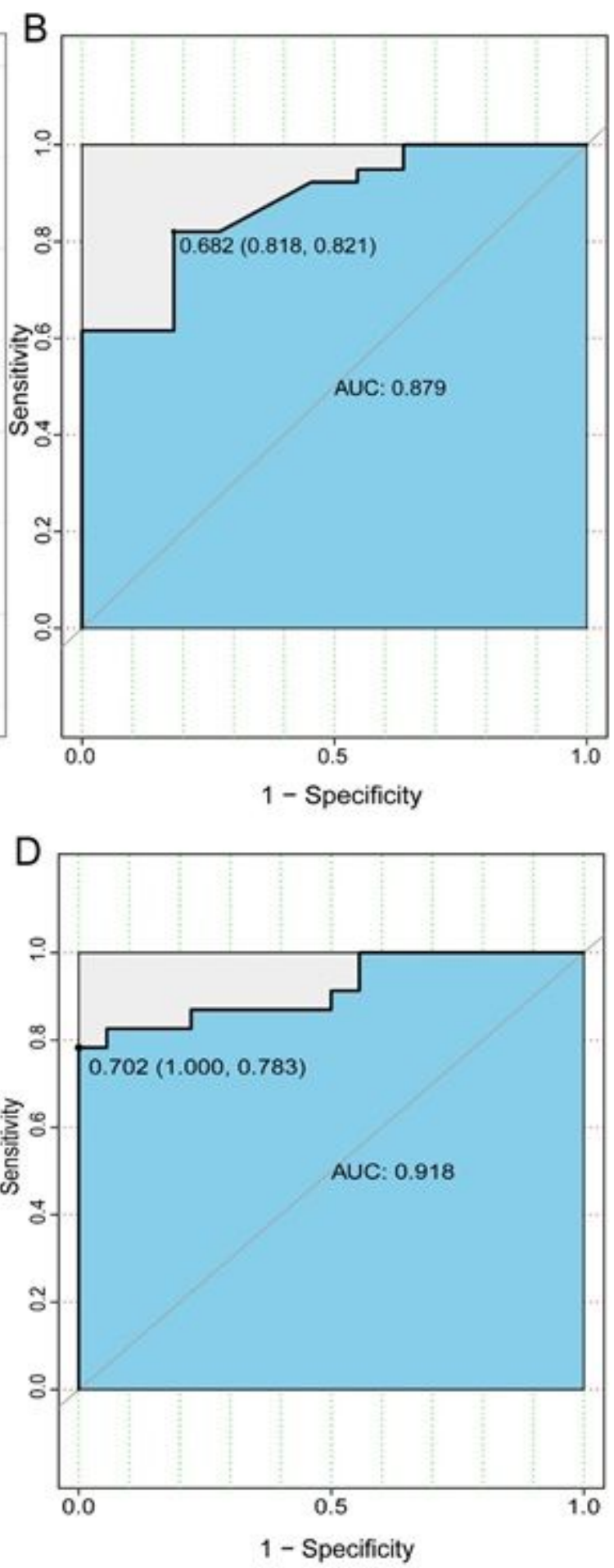

\section{Figure 2}

IL10RB expression according to different glioma subtypes in TCGA and CGGA datasets. IL10RB expression in four subtypes of glioma ( $A$ and $C$ ). Evaluating the efficacy of IL10RB in distinguishing the mesenchymal subtype from the three other subtypes by using ROC curve analysis ( $B$ and $D$ ). 

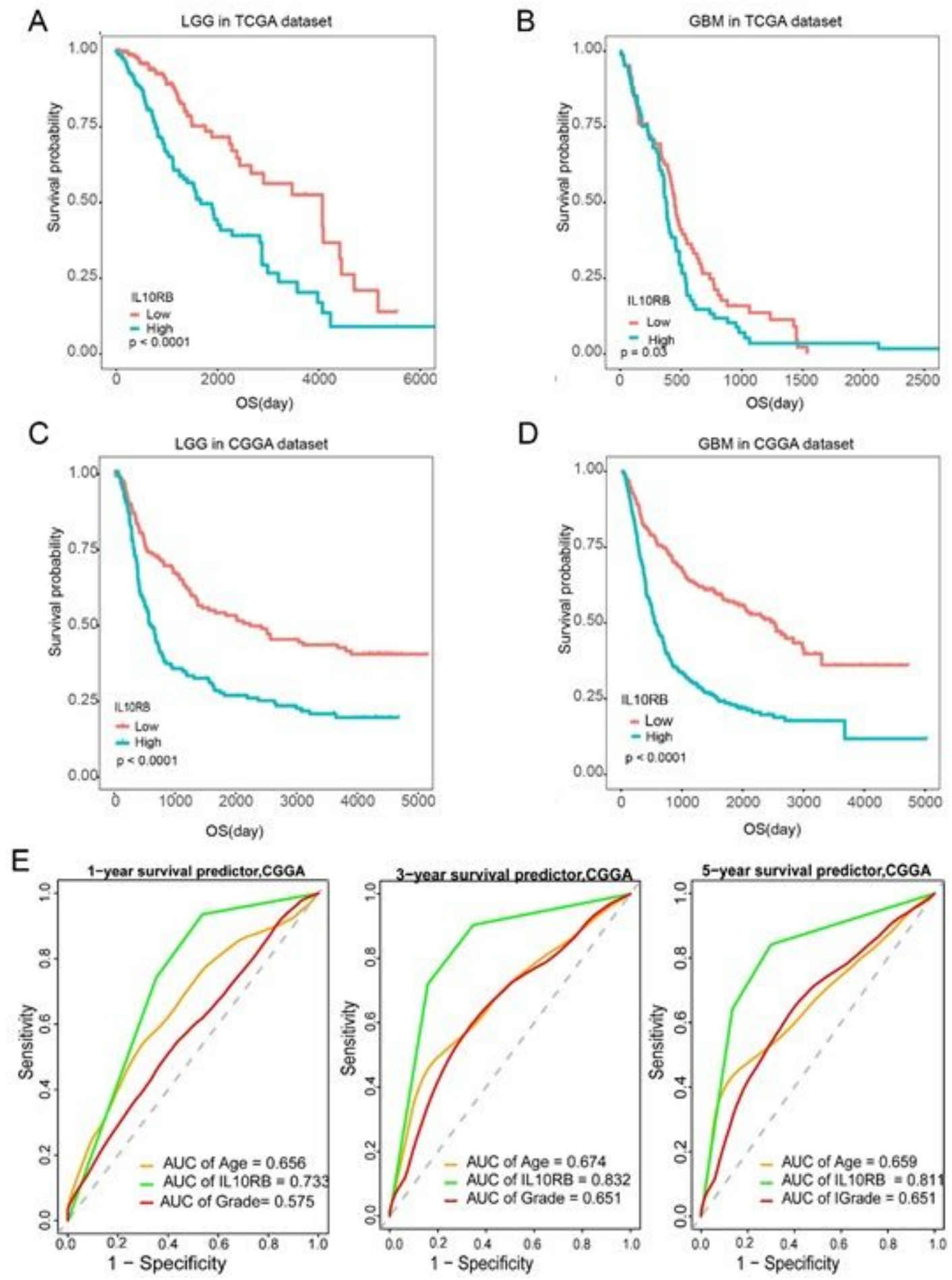

\section{Figure 3}

Kaplan-Meier survival analysis of IL10RB expression in glioma. Survival analysis of different IL10RB expression levels in LGG in the TGGA and CCGA datasets (A and C). Survival analysis of different IL10RB expression levels in GBM in the TGGA and CCGA datasets (B and D). Time-dependent ROC curve analysis of the sensitivity and specificity of IL10RB expression, age at diagnosis, and tumor grade in predicting 1-, 3-, and 5-year OS in the CGGA data set (E). 
A

Univariable Cox analysis in TCGA

$\begin{array}{ll}\text { Variable } & \text { P value } \\ \text { Gender } & 0.175 \\ \text { Age } & 0.007 \\ \text { Grade } & 0.003 \\ \text { Subtype } & 0.050 \\ \text { IDH1/2 mutation status } & 0.017 \\ \text { 1p/9q codeletion status }<0.001 \\ \text { MGMT promoter status } & 0.008 \\ \text { Radiotherapy } & 0.001 \\ \text { Chemotherapy } & 0.294 \\ \text { IL10RB } & 0.005\end{array}$

$\mathrm{HR}(95 \% \mathrm{Cl})$

$1.438(0.851-2.431)$

1.070(0.964-1.187)

$1.136(1.010-1.278)$

$0.105(0.932-1.309)$

2.644(0.784-8.922)

2.532(1.542-4.156)

$0.593(0.092-3.797)$

$0.343(1.127-1.602$

$0.957(0.769-1.191)$

$0.513(0.174-1.508)$

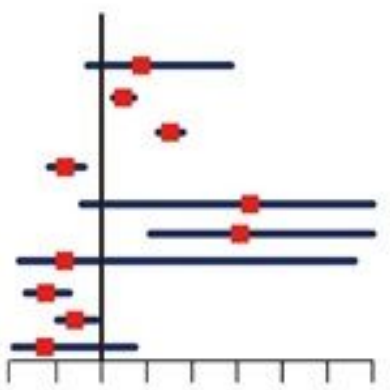

00.511 .522 .533 .54

Hazard ratio

B

\section{Multivariable Cox analysis in TCGA}

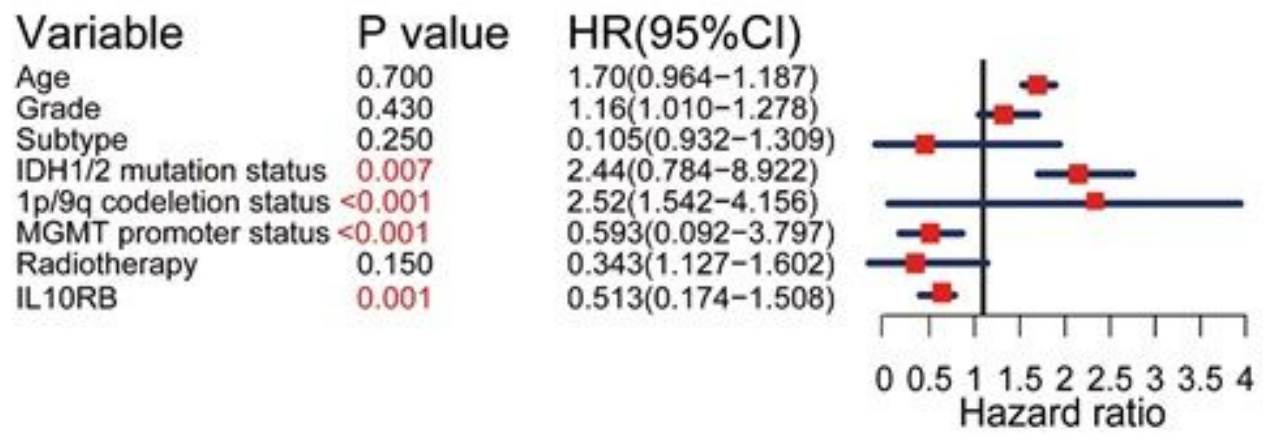

Figure 4

Univariable and multivariable Cox analysis of IL10RB expression, age, grade subtype, IDH1/2 mutation status, $1 p / 19 q$ codeletion status, MGMT promoter status, radiotherapy in the TCGA dataset.
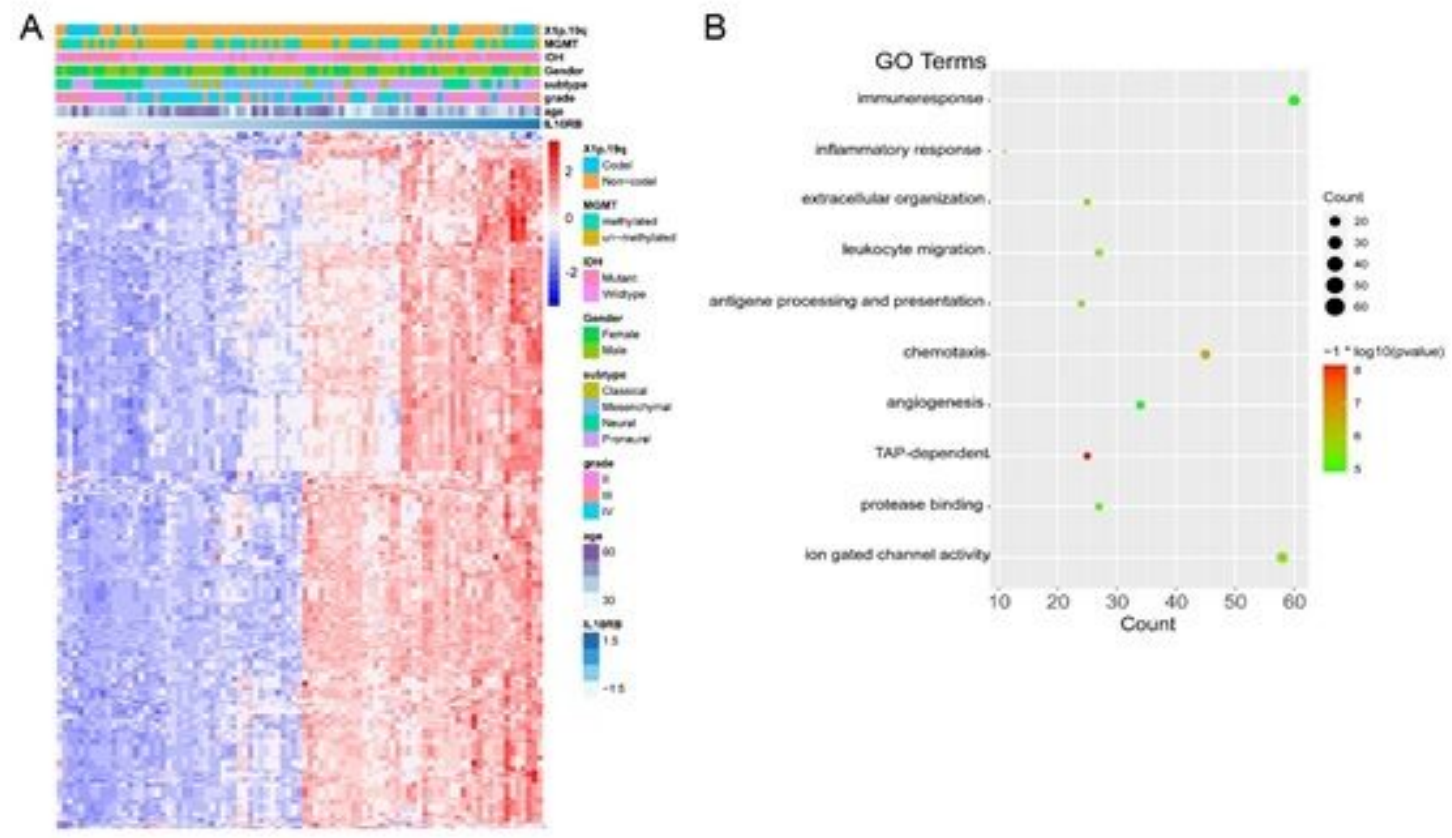


\section{Figure 5}

Genes positively related $(R>0.5)$ with IL10RB expression $(A)$, and the BP analysis results show that IL10RB expression is related to the immune function of gliomas (B) based on the TCGA dataset.

A TCGA
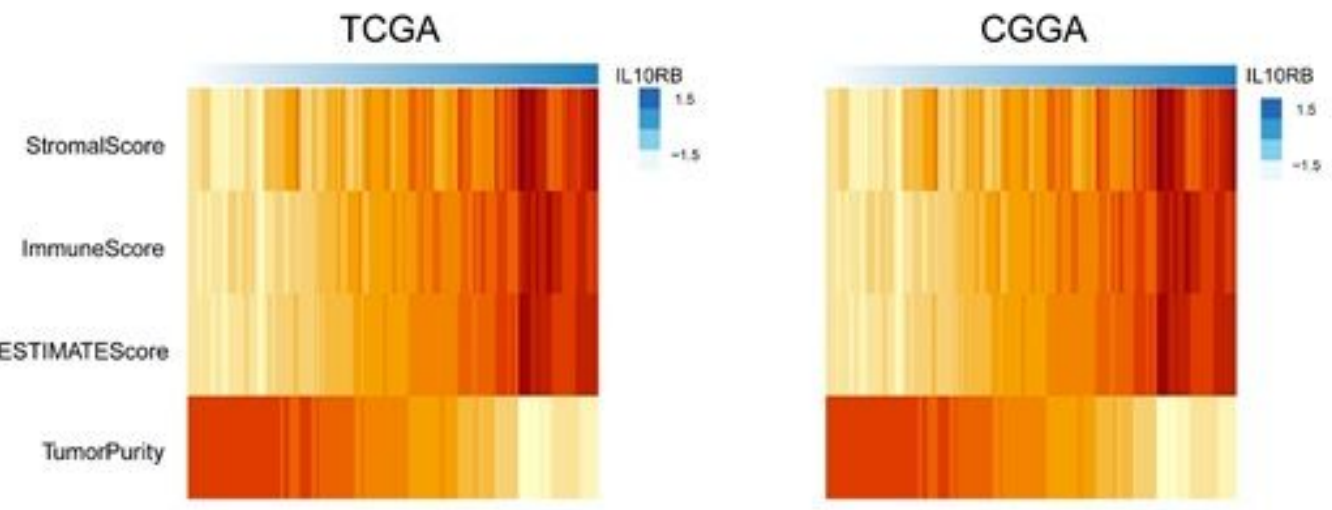

B TCGA

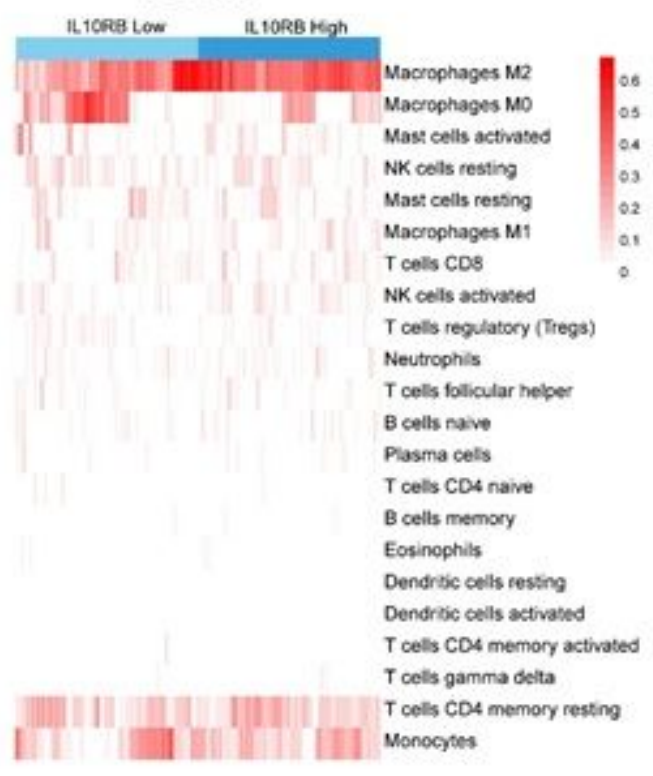

CGGA

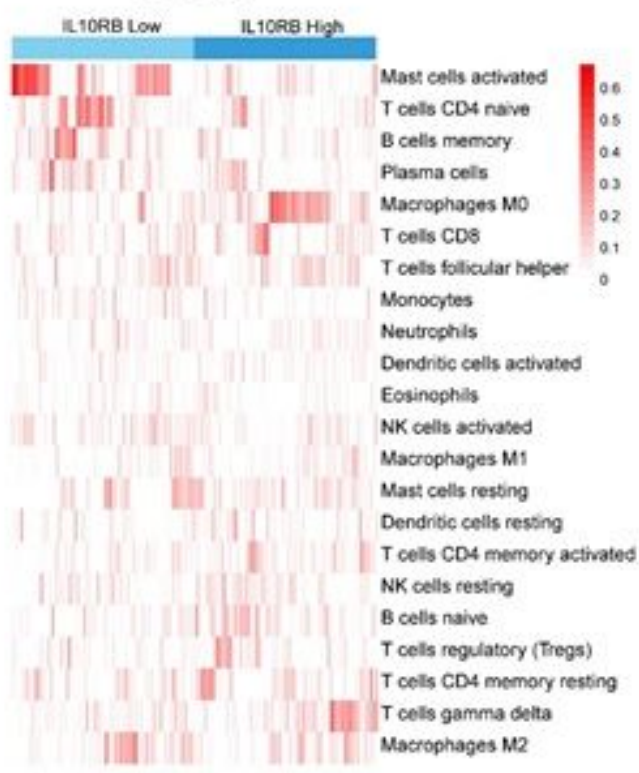

\section{Figure 6}

Inflection of high IL10RB expression on the immune environment. The immune score, tumor purity, stromal score, and ESTIMATE score under different IL10RB expression levels in the CGGA and TCGA datasets (A). Levels of multiple infiltrated immune cells under low or high IL10RB expression in the CGGA and TCGA datasets $(B)$. 
A

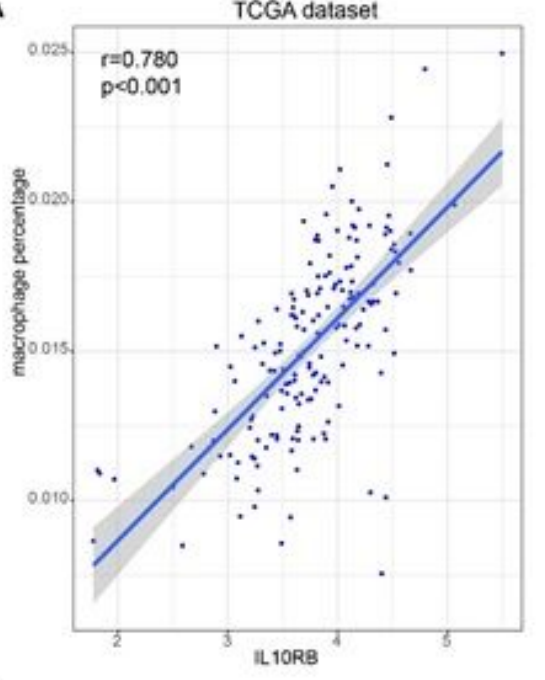

B

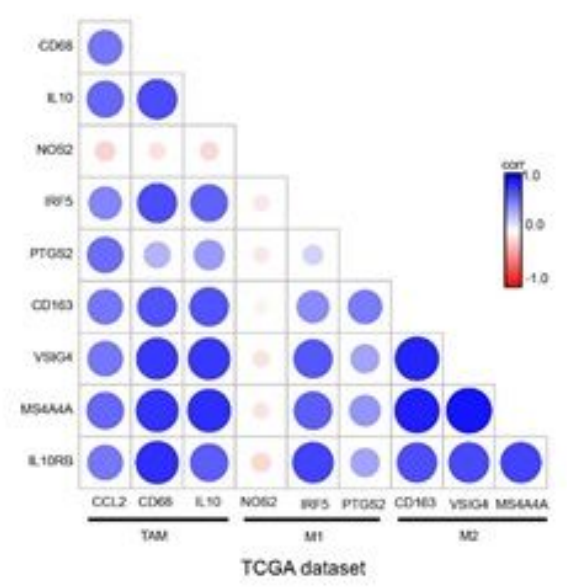

C

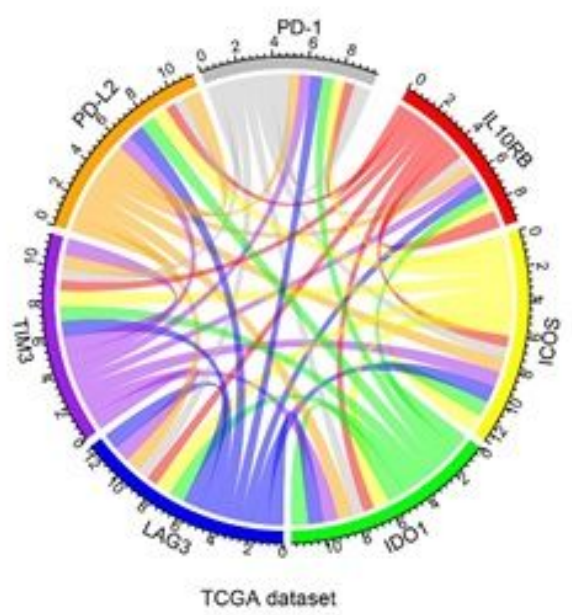

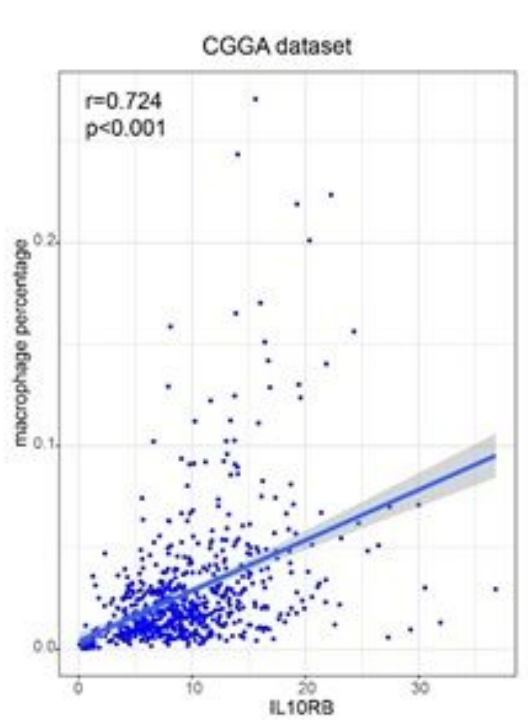
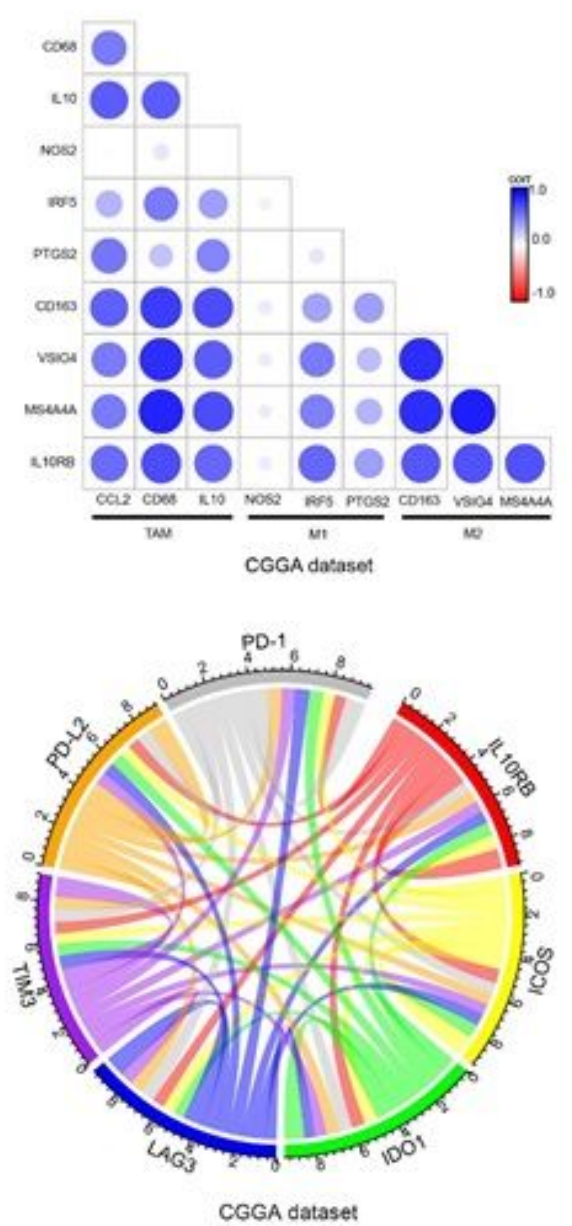

\section{Figure 7}

Strong correlation between IL10RB and immunosuppressive microenvironment. IL10RB had a strong positive correlation with macrophages (A). A positive correlation of IL10RB with TAMs and M2, not M1 in the TCGA and CGGA dataset (B). A positive correlation between IL10RB and checkpoints inhibiting immunity in the TCGA and CGGA dataset (C). 


\section{Supplementary Files}

This is a list of supplementary files associated with this preprint. Click to download.

- supplementarymaterials.docx 